\title{
Cellular Uptake Mechanism of Paclitaxel Nanocrystals Determined by Confocal Imaging and Kinetic Measurement
}

\author{
Yan Chen ${ }^{1,2}$ and Tonglei $\mathrm{Li}^{2,3}$
}

Received 12 March 2015; accepted 20 April 2015; published online 24 June 2015

\begin{abstract}
Nanocrystal formulation has become a viable solution for delivering poorly soluble drugs including chemotherapeutic agents. The purpose of this study was to examine cellular uptake of paclitaxel nanocrystals by confocal imaging and concentration measurement. It was found that drug nanocrystals could be internalized by KB cells at much higher concentrations than a conventional, solubilized formulation. The imaging and quantitative results suggest that nanocrystals could be directly taken up by cells as solid particles, likely via endocytosis. Moreover, it was found that polymer treatment to drug nanocrystals, such as surface coating and lattice entrapment, significantly influenced the cellular uptake. While drug molecules are in the most stable physical state, nanocrystals of a poorly soluble drug are capable of achieving concentrated intracellular presence enabling needed therapeutic effects.
\end{abstract}

KEY WORDS: cellular uptake; confocal microscopy; nanocrystals; paclitaxel; pharmacokinetics.

\section{INTRODUCTION}

Our in vivo experiments have demonstrated that nanocrystal formulations of chemotherapeutic agents are capable of eliciting similar and better anticancer efficacy-compared with solubilized or encapsulated delivery systems-but exhibiting much reduced side effects $(1,2)$. This may be contributed by the absence of helper chemicals that are used in the conventional formulations for solubilizing and/or encapsulating poorly soluble drugs (3-7). Any side effects associated with carrier chemicals are completely eliminated in (pure) nanocrystal formulations. Formulated as nanosized particles, a chemotherapeutic drug may also take advantage of possible leaky vasculatures found in tumor and augment local drug retention, a hallmark of the EPR (enhanced permeability and retention) effect. Importantly, nanocrystals are physically stable-albeit possible particle aggregation, which can be remedied or minimized by surface coating with surfactants - and unlikely to undergo drastic phase transition and structural change. Our recent study of paclitaxel nanocrystals (PTX-NCs) showed that the delivery system

Electronic supplementary material The online version of this article (doi:10.1208/s12248-015-9774-0) contains supplementary material, which is available to authorized users.

\footnotetext{
${ }^{1}$ Department of Pharmaceutical Science, School of Pharmacy, Second Military Medical University, Shanghai, 200433, China.

${ }^{2}$ Department of Industrial and Physical Pharmacy, College of Pharmacy, Purdue University, 575 Stadium Mall Drive, West Lafayette, Indiana 47907, USA.

${ }^{3}$ To whom correspondence should be addressed. (e-mail: tonglei@email.uky.edu)
}

had similar but extended tumor accumulation with Taxol®, possibly due to the localization of drug nanocrystals (1). In addition, it was observed that more than $40 \%$ of injected nanocrystals were taken up by liver, considerably surpassing that by Taxol. It remains, however, enigmatic how drug nanocrystals exhibit anticancer effects to cancer cells, whether through dissolution followed by membrane diffusion into the cells or being taken up directly through endocytosis. It is further interesting to explore the impact by surface-coating nanocrystals with hydrophilic surfactants on the cellular uptake, shedding light on ways to improving pharmacokinetics and biodistribution of drug nanocrystal formulations and to particularly reduce possible liver toxicities due to significant uptake of drug nanocrystals by the mononuclear phagocyte system.

As such, the purpose of this report is to unveil cellular uptake mechanisms of drug nanocrystals and understand their therapeutic effects. Specifically, we tested in cells hybrid PTXNCs, in which a fluorescent dye was physically integrated $(2,8)$, as well as surface-coated nanocrystals with polymers and surfactants. Through chemical analyses and confocal imaging, it was found that the drug nanocrystals could be internalized by cells directly and at much higher concentrations than those in the cells treated by the solubilized formulation.

\section{EXPERIMENTAL PROCEDURES}

\section{Materials}

Paclitaxel (PTX) (>99.5\% purity) was purchased from LC Laboratories (Woburn, MA, USA). Sulforhodamine B 
(SRB), folic acid (FA), dopamine, Pluronic $\AA \mathrm{F}_{68}$, and $\mathrm{F}_{127}$ were obtained from Sigma-Aldrich Inc. (St. Louis, MO, USA). FA$\mathrm{PEG}_{3400}-\mathrm{NH}_{2}, \mathrm{mPEG}_{2000^{-}}-\mathrm{NH}_{2}$, fluorescein(FITC)-PEG $\mathrm{P}_{2000^{-}}$ $\mathrm{NH}_{2}$, and $\mathrm{mPEG}-\mathrm{FITC}$ were purchased from Nanocs Inc. (New York, NY, USA). Hoechst 33258 and Lysotracker ${ }^{\circledR}$ Deep Red were obtained from Life Technologies Inc. (Grand Island, NY, USA).

\section{Preparation of PTX Nanocrystals}

PTX-NCs were grown from solution by an anti-solvent crystallization method reported in our previous studies (8). In general, $1 \mathrm{~mL}$ of $3 \mathrm{mg} / \mathrm{mL}$ PTX dissolved in ethanol was introduced into $20 \mathrm{~mL}$ of deionized water in a flask. The solution was sonicated and under high-speed stirring. The suspension was then filtered with a 50-nm polycarbonate filter paper and the retentate was re-suspended in deionized water by homogenization.

\section{Preparation of Fluorescein Hybrid Paclitaxel Nanocrystals}

To produce SRB-integrated hybrid nanocrystals, the same procedure was used but with the dye dissolved in the deionized water prior to the crystallization process (8). After crystallization and filtration, the retentate was re-suspended in water and underwent additional centrifugation-resuspension cycles to remove any loosely bounded dye molecules on the surface of nanocrystals. PEG and FAPEG-integrated PTX hybrid nanocrystals were also prepared with the same approach where PEG or FA-PEG was present in the crystallization medium.

\section{Preparation of PEG Hybrid Paclitaxel Crystals}

To verify the possibility of PEG integration in the crystal lattice of PTX, larger hybrid crystals were prepared with a similar procedure but without sonication and high-speed stirring. Briefly, $1 \mathrm{~mL}$ of $3 \mathrm{mg} / \mathrm{mL}$ paclitaxel in ethanol was added into $20 \mathrm{~mL}$ of deionized water in vial and, subsequently, FITC-PEG was introduced to the solution. The FITCPEG-PTX hybrid crystals were collected by centrifugation-resuspension cycles for three times. The crystals were observed by a confocal microscope (Nikon A1) at $488 \mathrm{~nm}$.

\section{Preparation of Surface-Treated PTX Nanocrystals}

PTX-NCs were treated with several different polymers in order to modify the surface chemistry of the nanocrystals. Polydopamine(Dp)-coated nanocrystals were prepared according to the reported method with some modification $(9,10)$. Three milligrams PTX nanocrystals was added into $20 \mathrm{~mL}$ of Tris- $\mathrm{HCl}$ solution $(10 \mathrm{mmol} / \mathrm{L}, \mathrm{pH}=8.5)$ under sonication. Dopamine was added into the mixture and self-polymerization was allowed to proceed for $3 \mathrm{~h}$ at the room temperature. The Dp-PTX-NCs were collected by centrifugation. In addition, Dp-PTX-NCs were dispersed in PBS solution (1 $\mathrm{mmol} / \mathrm{L}, \mathrm{pH}=7.4)$ under sonication and then incubated with $2 \mathrm{mg} / \mathrm{mL}$ FA-PEG $3400-\mathrm{NH}_{2}$ for $2 \mathrm{~h}$ at the room temperature. The FA-PEG-Dp-PTX-NCs were collected by centrifugation. PEG-Dp-PTX-NCs were prepared with the same method but with $2 \mathrm{mg} / \mathrm{mL} \mathrm{mPEG}_{2000}-\mathrm{NH}_{2}$ instead.
To verify the surface-coating technique, larger crystals of PTX were prepared with SRB integrated in the crystal in accordance with the similar crystallization method for preparing hybrid nanocrystals. Briefly, $1 \mathrm{~mL}$ of $3 \mathrm{mg} / \mathrm{mL}$ paclitaxel in ethanol was added into $20 \mathrm{~mL}$ of deionized water with SRB in the vial without stirring and sonication. The SRB-PTX crystals were then treated with polydopamine as described above and then conjugated with FITC-PEG ${ }_{2000}-\mathrm{NH}_{2}$. The FITC-PEGSRB-PTX crystals were obtained by three centrifugation-resuspension cycles to remove loosely bounded polymers and fluorescent molecules from the surface of the crystals. The crystals were observed by a confocal microscope (Nikon A1).

Physically coated PTX-NCs were prepared by treating nanocrystals with Pluronics $\mathrm{F}_{68}$ and $\mathrm{F}_{127}$. Three milligrams PTX nanocrystals was re-suspended in 3-mL deionized water under sonication. Two percent $(\mathrm{m} / \mathrm{m})$ Pluronics $\mathrm{F}_{68}$ or $\mathrm{F}_{127}$ was added to the solution under mechanical shaking for $3 \mathrm{~h}$ at $4^{\circ} \mathrm{C} . \mathrm{F}_{68} / \mathrm{F}_{127^{-}}$ PTX-NCs were collected by centrifugation-washing cycles for three times to remove loosely bounded polymers.

\section{Characterization of PTX Nanocrystals}

Particle size and zeta potential of nanocrystals were determined by dynamic light scattering (DLS) (Zetasizer Nano ZS, Malvern) at $25^{\circ} \mathrm{C}$. Each measurement was done in triplicate. Particle morphology was detected by a scanning electron microscope (SEM, Nova NanoSEM, FEI) at an accelerating voltage of $5 \mathrm{kV}$. The samples were completely dried under vacuum and coated with conductive layers of gold palladium $(\mathrm{Au} / \mathrm{Pd})$ for $1 \mathrm{~min}$ at a current of $20 \mathrm{~mA}$ prior to the SEM measurement.

Chemical quantification of PTX was determined by a highpressure liquid chromatography (HPLC, Agilent 1100) with a C18 column $(5 \mu \mathrm{m}, 4.6 \times 150 \mathrm{~mm})$ and a UV detector at $227 \mathrm{~nm}$. The mobile phase of acetonitrile and water $(50: 50 \mathrm{v} / \mathrm{v})$ were pumped at a total flow rate of $1 \mathrm{~mL} / \mathrm{min}$. The column was kept in $25^{\circ} \mathrm{C}$ and the injection volume of samples was $20 \mu \mathrm{L}$.

\section{Cell Culture}

Human KB nasopharyngeal epidermal carcinoma cells were obtained from ATCC (Manassas, VA, USA). Cells were cultured in folic acid (FA)-deficient RPMI1640 medium supplemented with $10 \%$ fetal bovine serum, $100 \mathrm{U} / \mathrm{mL}$ penicillin and $100 \mu \mathrm{g} / \mathrm{mL}$ streptomycin. The cell culture was done at $37^{\circ} \mathrm{C}$ in a humidified atmosphere containing $5 \% \mathrm{CO}_{2}$.

\section{Cellular Uptake Imaging of Nanocrystals}

$\mathrm{KB}$ cells were seeded in confocal petri dishes at a density of $2 \times 10^{5}$ cells/well and cultured for $24 \mathrm{~h}$. After adhering to dish walls, the KB cells were incubated in FA-deficient RPMI 1640 medium with $100 \mu \mathrm{g} / \mathrm{mL}$ SRB-PTX-NCs at different durations $(15,30 \mathrm{~min}$, 1,2 , and $3 \mathrm{~h}$ ) or incubated for $3 \mathrm{~h}$ with the concentration of the nanocrystals varied $(1,5,25$, and $100 \mu \mathrm{g} / \mathrm{mL})$. Additionally, the $\mathrm{KB}$ cells were incubated for $3 \mathrm{~h}$ in FA-deficient RPMI1640 medium with $100 \mu \mathrm{g} / \mathrm{mL}$ bare or surface-modified SRB-PTX-NCs (PEG-Dp-SRB-PTX-NCs, FA-PEG-Dp-SRB-PTX-NCs, PEGSRB-PTX-NCs, FA-PEG-SRB-PTX-NCs, F ${ }_{127}$-SRB-PTX-NCs, and $\mathrm{F}_{68}$-SRB-PTX-NCs). At the end of incubation, cells were gently washed with cold PBS for three times. Lysotracker Red 
(red) was added at the concentration of $100 \mathrm{nM}$ and the cells were further incubated at $37^{\circ} \mathrm{C}$ for $30 \mathrm{~min}$. After being collected and washed, the cells were fixed with $4 \%$ paraformaldehyde in PBS at the room temperature for $10 \mathrm{~min}$, followed by cell nuclei staining with $2 \mathrm{~mL}$ Hoechst $33258(2 \mu \mathrm{g} / \mathrm{mL})$ for $15 \mathrm{~min}$ (blue). The cells were imaged by a laser scanning confocal microscope (Nikon A1).

\section{Cellular Uptake Kinetics}

$\mathrm{KB}$ cells were treated with nanocrystals and drug concentrations in the medium and inside cells were analyzed by HPLC. $\mathrm{KB}$ cells were seeded in 6-well plates at a density of $2 \times 10^{5}$ cells/ well and cultured for $24 \mathrm{~h}$. Upon adhesion, the KB cells were incubated in FA-deficient RPMI 1640 medium with $100 \mu \mathrm{g} / \mathrm{mL}$ pure PTX-NCs and Taxol, respectively, for different durations $(0.5,1,2$, and $3 \mathrm{~h})$ or for $3 \mathrm{~h}$ with different drug concentrations $(12.5,25,50$, and $100 \mu \mathrm{g} / \mathrm{mL})$. Temperature effect was studied by incubating with the drug formulations in 37 and $4{ }^{\circ} \mathrm{C}$, respectively. Influence of FBS (fetal bovine serum) on cellular uptake was also studied by treating KB cells with $100 \mu \mathrm{g} / \mathrm{mL}$ pure PTXNCs and 0, 10, and 20\% FBS, respectively, in FA-deficient RPMI 1640 medium. In addition, polymer-treated nanocrystals were used for treating $\mathrm{KB}$ cells at $25 \mu \mathrm{g} / \mathrm{mL}$ in FA-deficient RPMI1640 medium for $3 \mathrm{~h}$. At the end of incubation, $1 \mathrm{~mL}$ of the culture medium was collected and centrifuged at 10,500 rpm for $15 \mathrm{~min}$. The supernatant, $0.5 \mathrm{~mL}$, was removed and analyzed for the extracellular solubilized drug concentration. The rest of the culture medium was collected and cells were washed three times with cold PBS. The total extracellular drug concentration, which is of both the extracellular solubilized drug and the extracellular drug nanocrystals, was then obtained from the analyses of the remaining liquid in the centrifugation tube, the rest of the culture medium, and the PBS liquid used for washing the cells, in addition to the concentration already determined from the supernatant. The cells were treated with $0.25 \%$ trypsinEDTA and lysed by a Selecta Sonopuls (Nikon A1, Nikon Co. Ltd., Tokyo, Japan) for determining the drug concentration (i.e., total intracellular drug concentration). All samples were diluted properly with acetonitrile and PTX was quantified by HPLC.

The Taxol formulation used in treating cells was prepared by dissolving PTX into a 50:50 mixture of ethanol and Cremophor EL at room temperature.

\section{Statistical Analyses}

Cellular uptake data were represented as the mean with standard deviation (SD). One-way analysis of variance (ANOVA) was performed to determine the null hypothesis among groups, followed by Bonferroni post hoc test. All statistical tests were two-sided, and $P$ values less than 0.05 were considered to be statistically significant.

\section{RESULTS AND DISCUSSION}

\section{Characterization of PTX Nanocrystals}

Particle sizes of PTX-NCs were measured by DLS. Immediately after the crystallization process, PTX-NCs showed a wider particle size distribution ranging with a major peak at $300 \mathrm{~nm}$ and a small one at $5441 \mathrm{~nm}$ (Figure S1A in
Supporting Information). The distribution narrowed to 100 $500 \mathrm{~nm}$ with one major peak at $239 \mathrm{~nm}$ (Figure S1B), illustrating the effect of high-speed homogenization.

Table I lists DLS measurements of particle size and surface potential of the drug nanocrystals prepared in the study. The polydopamine had little effect on the particle size of PTX nanocrystals but negated the zeta potential significantly (Dp-PTX-NCs); PEG conjugation (PEG-Dp-PTXNCs) showed little additional effect, but FA-PEG (FA-PEGDp-PTX-NCs) seemed to neutralize the dopamine's effect to some extent. The Pluronics $\mathrm{F}_{68^{-}}$and $\mathrm{F}_{127}$-coated nanocrystals had similar particle size ranges and zeta potentials as pure PTX-NCs. For the hybrid nanocrystals, both PEG and SRB integration (PEG-PTX-NCs and SRB-PTX-NCs) showed minor influence, but FA-PEG integration (FA-PEG-PTXNCs) enlarged the nanocrystals greatly to more than $800 \mathrm{~nm}$ in average size and also changed the sign of zeta potential from negative to positive. It is suspected that the polymer (FA-PEG-NH $\mathrm{N}_{2}$ ) on the surface of the nanocrystals might induce some degree of aggregation during the DLS measurement because SEM showed much smaller crystals.

PTX-NCs appeared to be rod-like as observed by SEM (Fig. 1a). The longest dimension of the nanocrystals generally agrees with the particle size measured by DLS. The polymercoated or integrated nanoparticles show similar rod-shape morphologies (Fig. 1c-f), suggesting that the surface treatment or lattice integration by the polymers had little influence on the particles. Again, the FA-PEG-PTX-NCs appeared to be aggregated to some extent but individual crystals were mostly smaller than $500 \mathrm{~nm}$ (Fig. 1e). On the other hand, the integration of SRB forced the crystals (SRB-PTX-NCs) to grow more elongated (Fig. 1b).

To verify the experimental procedure that permits amino-terminated PEG-based polymers to be conjugated to dopamine-coated crystals, confocal imaging was used to observe co-localization of the FITC-PEG 2000 coating and Dp-SRB-PTX crystals (Fig. 2). On superimposing fluorescent images of FITC and SRB channels, both chemicals, FITC$\mathrm{PEG}_{2000}$ and $\mathrm{SRB}$, were present with the crystals suggesting that the polymer was linked to the crystals through the polydopamine coating. In addition, Fig. 2d hybrid PTX crystals in which FITC-PEG 2000 was physically integrated, supporting the crystallization method to physically incorporate polymers into the drug crystal.

Table I. Particle Size and Zeta Potential of PTX Nanocrystals $($ Mean \pm SD; $n=3)$

\begin{tabular}{lccc}
\hline & $\begin{array}{c}\text { Particle } \\
\text { size }(\mathrm{nm})\end{array}$ & $\begin{array}{c}\text { Polydispersity } \\
\text { index }\end{array}$ & $\begin{array}{c}\text { Zeta potential } \\
(\mathrm{mV})\end{array}$ \\
\hline PTX-NCs & $240 \pm 11$ & $0.16 \pm 0.06$ & $-10.7 \pm 1.1$ \\
Dp-PTX-NCs & $231 \pm 11$ & $0.08 \pm 0.05$ & $-27.5 \pm 1.3$ \\
PEG-Dp-PTX-NCs & $235 \pm 17$ & $0.12 \pm 0.04$ & $-28.5 \pm 0.8$ \\
FA-PEG-Dp-PTX- & $233 \pm 4$ & $0.10 \pm 0.02$ & $-16.5 \pm 0.9$ \\
$\quad$ NCs & & & \\
PEG-PTX-NCs & $276 \pm 52$ & $0.10 \pm 0.08$ & $-9.0 \pm 0.3$ \\
FA-PEG-PTX-NCs & $825 \pm 151$ & $0.43 \pm 0.35$ & $6.1 \pm 0.8$ \\
F $_{68}$-PTX-NCs & $334 \pm 23$ & $0.19 \pm 0.05$ & $-8.3 \pm 0.6$ \\
F $_{127}$-PTX-NCs & $234 \pm 21$ & $0.06 \pm 0.03$ & $-5.9 \pm 0.1$ \\
SRB-PTX-NCs & $243 \pm 22$ & $0.16 \pm 0.04$ & $-12.9 \pm 0.9$ \\
\hline
\end{tabular}



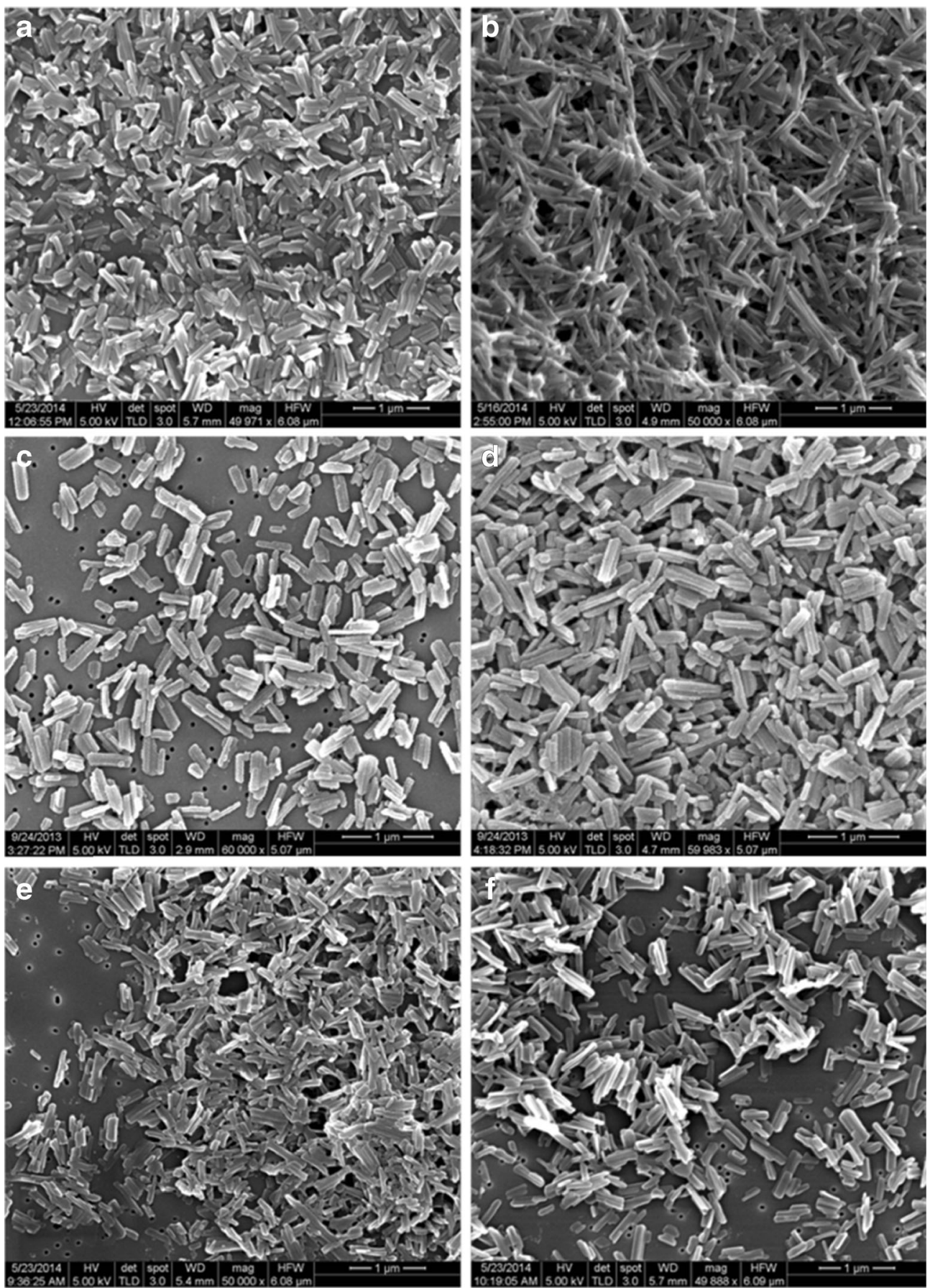

Fig. 1. SEM micrographs of a PTX-NCs, b SRB-PTX-NCs, $\mathbf{c}$ Dp-PTX-NCs, d FA-PEG-Dp-PTX-NCs, e FA-PEG-PTX-NCs, and $\mathbf{f} \mathrm{F}_{68}$-PTX-NCs. Scale bar, $1 \mu \mathrm{m}$

\section{Confocal Imaging of Cellular Uptake of Drug Nanocrystals}

Cellular uptake of paclitaxel nanocrystals that physically integrated SRB (SRB-PTX-NCs) was observed by confocal microscopy. The concentration of SRB in the nanocrystals was determined less than $2 \%(w / w)$ by fluorospectrometry. No fluorescence was observed inside KB cells when cocultured with SRB solution (Figure S2 in Supporting Information), indicating that free dye molecules could not be taken up significantly by the cells. This may be due to the anionic nature of the dye that limits the molecules across cell membranes. Conversely, significant SRB intensities were observed intracellularly, especially when the centration of nanocrystals in the culture medium was greater than $25 \mu \mathrm{g} /$ $\mathrm{mL}$ (Fig. 3). The internalization of SRB implies that nanocrystals were directly taken up by the cells such that the fluorescence could only be emitted by the dye molecules that were carried into the cells by the nanocrystals, either integrated within the nanocrystals or dissolved inside the cells. Moreover, the co-localization (orange) of green and red intensities of fluorescence points out endocytosis responsible for the cellular uptake of the nanocrystals. Figure 4 further 

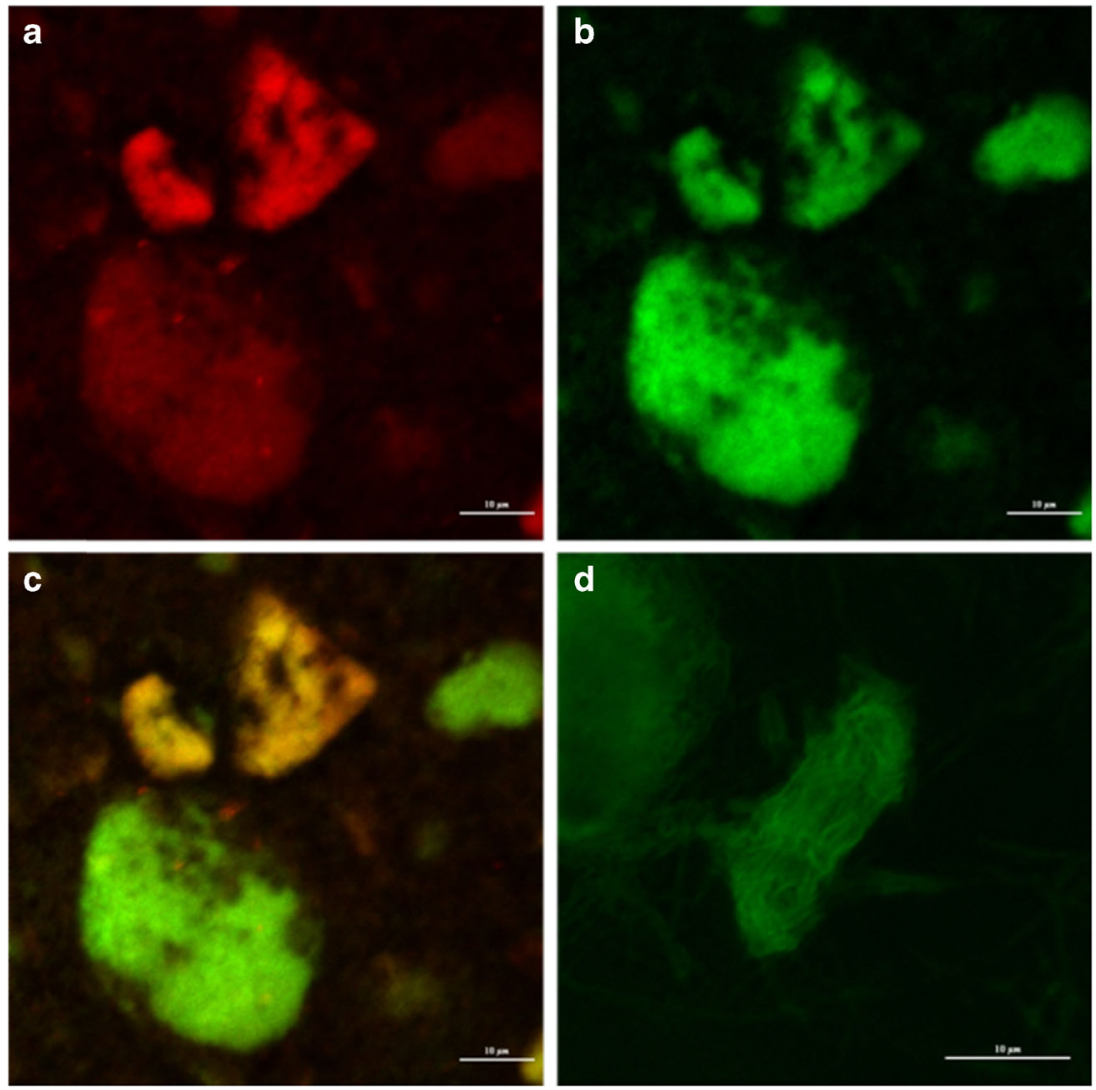

Fig. 2. Confocal images of FITC-PEG ${ }_{2000}$ coated Dp-SRB-PTX crystals observed at the a SRB channel, b FITC channel, and $\mathbf{c}$ both channels superimposed. Paclitaxel crystals where FITC-PEG ${ }_{2000}$ was physically incorporated is observed in the $\mathbf{d}$ FITC channel. Scale bar, $10 \mu \mathrm{m}$

illustrates cellular uptake as a function of time. At $15 \mathrm{~min}$, noticeable amounts of SRB-PTX-NCs were seen inside the cells and the fluorescence intensified as the incubation time increased. The cellular uptake of drug nanocrystals thus appeared to be rapid.

Celluar uptake of polymer-treated drug nanocrystals were studied by confocal microscopy in a similar fashion. All treated groups emitted green fluorescence in the KB cells, indicating direct cellular uptake of the drug nanocrystals (Fig. 5). Co-localization of SRB signals (green) and lysosomes (red) was observed by all the groups, revealing the nanocrystal uptake by endocytosis. Some of the nanocrystals appeared to be attached to the peripheral of KB cells. Among the groups, PEG or FA-PEG-conjugated nanocrystals (PEGDp-SRB-PTX-NCs and FA-PEG-Dp-SRB-PTX-NCs) showed no enhanced uptake (Fig. 5b, c), compared with the untreated group (SRB-PTX-NCs, Fig. 5a). The two hybrid nanocrystals (PEG-SRB-PTX-NCs and FA-PEG-SRB-PTXNCs), where PEG-based polymers were physically integrated into the nanocrystals during the preparation process, exhibited strong intracellular fluorescence due to SRB (Fig. 5d, e). For the two PEG-coated nanocrystals $\left(\mathrm{F}_{68}-\mathrm{SRB}-\mathrm{PTX}-\mathrm{NCs}\right.$

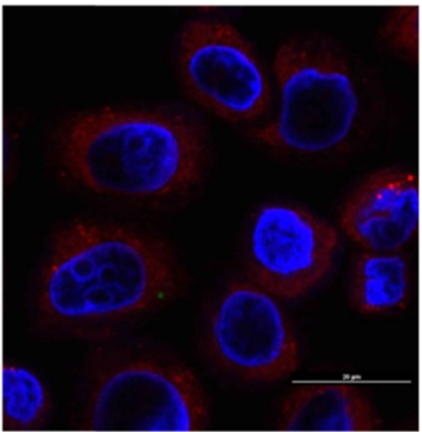

$1 \mu \mathrm{g} / \mathrm{mL}$

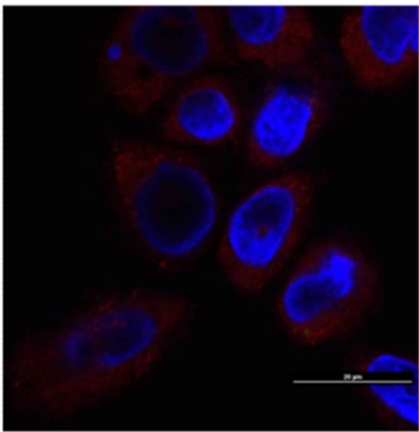

$5 \mu \mathrm{g} / \mathrm{mL}$

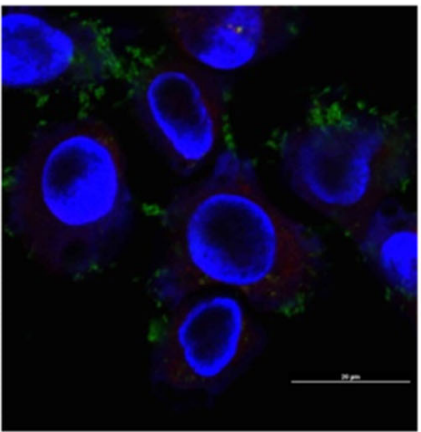

$25 \mu \mathrm{g} / \mathrm{mL}$

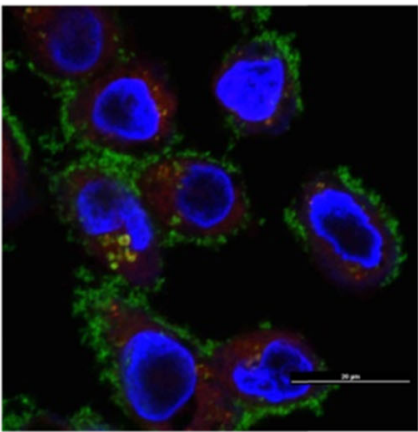

$100 \mu \mathrm{g} / \mathrm{mL}$

Fig. 3. Confocal images of KB cells treated by SRB-PTX-NCs of various concentrations at $3 \mathrm{~h}$. Nuclei and lysosomes were dyed as blue and red, respectively. Green was due to SRB. Scale bar, $10 \mu \mathrm{m}$ 


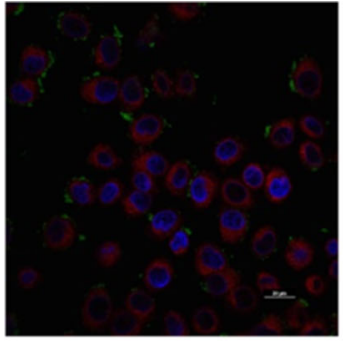

$15 \mathrm{~min}$

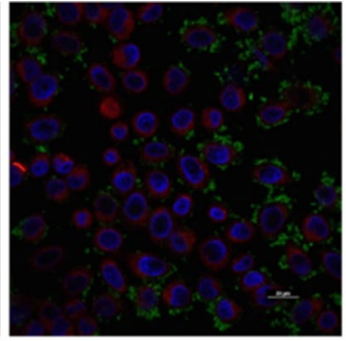

$30 \mathrm{~min}$

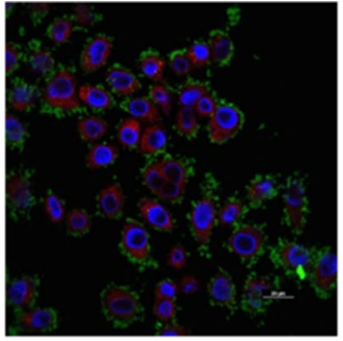

$1 \mathrm{~h}$

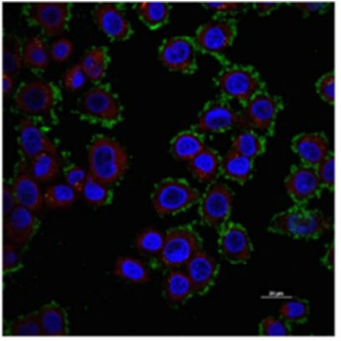

$2 \mathrm{~h}$

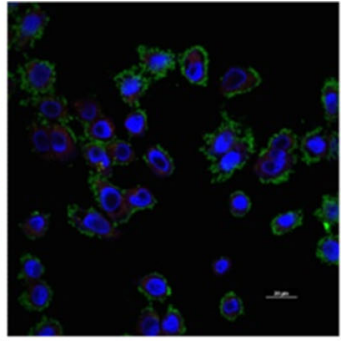

$3 \mathrm{~h}$

Fig. 4. Confocal images of KB cells treated by $100 \mu \mathrm{g} / \mathrm{mL}$ SRB-PTX-NCs at various time points. Nuclei and lysosomes were dyed as blue and $r e d$, respectively. Green was due to SRB. Scale bar, $20 \mu \mathrm{m}$

and $\mathrm{F}_{127}$-SRB-PTX-NCs), $\mathrm{F}_{68}$ seemed to promote additional crystal uptake (Fig. 5f), relative to $\mathrm{F}_{127}$-coated and untreated systems (Fig. 5a, g). The results unveil that, at least for KB cells, not only were the PTX nanocrystals directly internalized by the cells, but the polymer treatment (coating or lattice integration) could also have a profound impact. Interestingly, the FA-treated systems showed no direct enhancement of
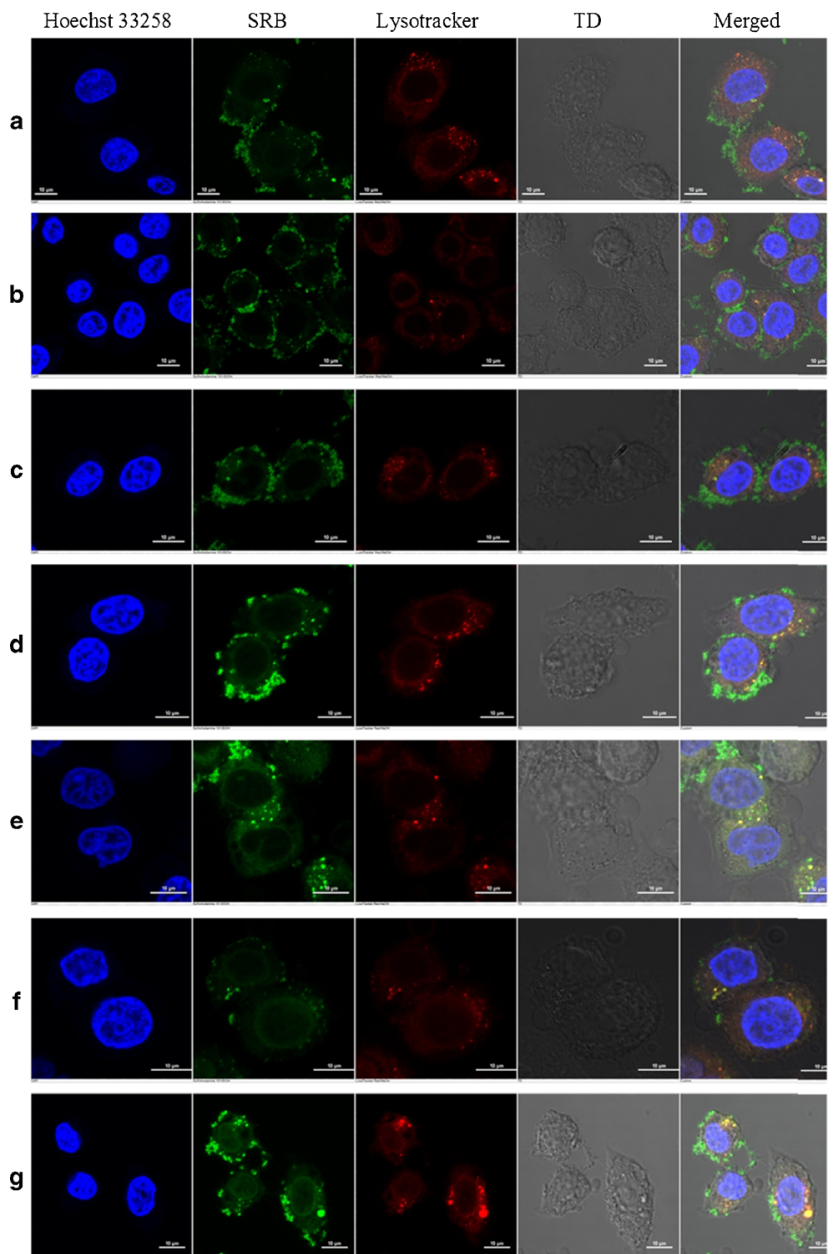

Fig. 5. Confocal images of KB cells treated by $100 \mu \mathrm{g} / \mathrm{mL}$ polymertreated nanocrystals at $3 \mathrm{~h}$, a SRB-PTX-NCs, b PEG-Dp-SRB-PTXNCs, c FA-PEG-Dp-SRB-PTX-NCs, d PEG-SRB-PTX-NCs, e FAPEG-SRB-PTX-NCs, f $\mathrm{F}_{127}$-SRB-PTX-NCs, and g $\mathrm{F}_{68}$-SRB-PTXNCs. Nuclei and lysosomes were dyed as blue and red, respectively. Green was due to SRB. Scale bar, $10 \mu \mathrm{m}$ cellular uptake, despite $\mathrm{KB}$ cells overexpressing folate receptors (Figure S3 in Supporting Information). It is suspected that FA moieties might not be sufficiently exposed on the crystal surface, whether via surface conjugation to the polydopamine-coated nanocrystals or physical integration to the drug crystal lattice.

\section{Cellular Uptake Kinetics}

In order to further characterize the cellular uptake of PTX nanocrystals, drug concentrations in the culture media and internalized within cancer cells were quantified by HPLC. Figure 6 shows respective drug concentrations of Taxol and PTX-NCs formulations in KB cells. For the Taxol group, less than $0.5 \%$ of the total delivered drug was found taken up by the cells, while the majority of the drug remained solubilized outside the cells. No significant changes in concentration were detected as the incubation prolonged. The nanocrystals, on the other hand, demonstrated drastically different kinetics. The amount of the drug found inside cells was significant, increased from about $15 \%$ at $0.5 \mathrm{~h}$ to more than $66 \%$ at $3 \mathrm{~h}$ of incubation. Accordingly, the drug concentration in the culture media decreased over time from more than $80 \%$ to less than

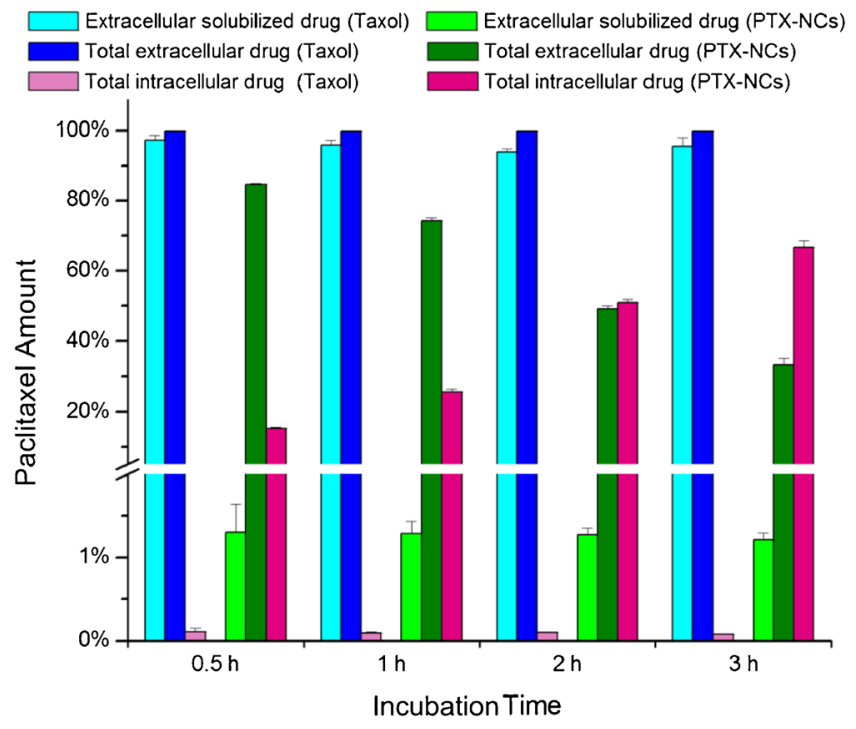

Fig. 6. Cellular uptake measurement of PTX in KB cells of Taxol (soluble PTX) and nanocrystals (PTX-NCs) at different incubation times. The total drug treatment was $100 \mu \mathrm{g} / \mathrm{mL}$ and intracellular and extracellular concentrations were measured 


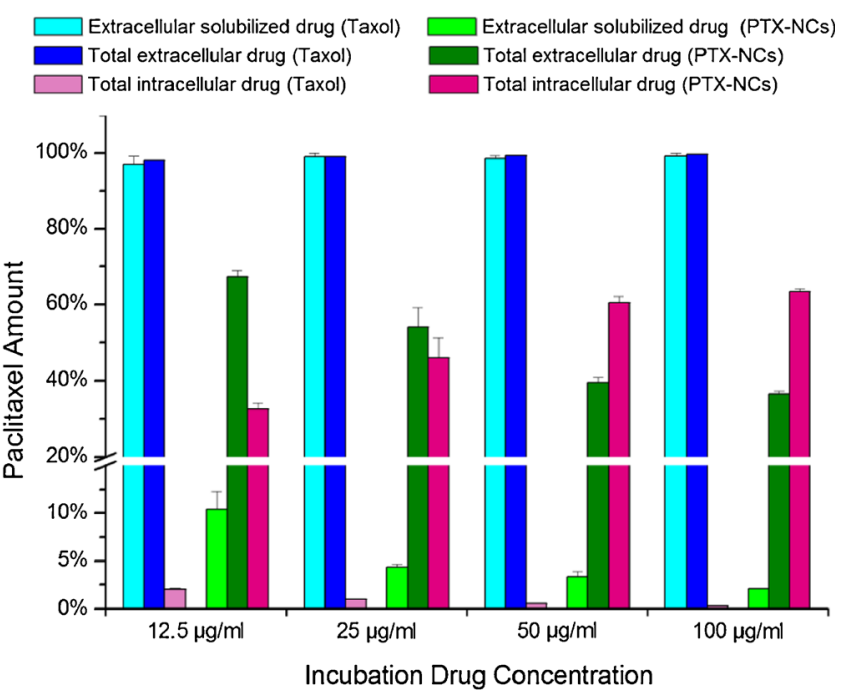

Fig. 7. Cellular uptake measurement of PTX in KB cells of Taxol (soluble PTX) and nanocrystals (PTX-NCs) at various concentrations. The incubation was $3 \mathrm{~h}$ and intracellular and extracellular drug concentrations were measured

$40 \%$. The solubilized drug outside the cells remained constant around $1.5 \%$, likely due to the solubilization equilibrium with the nanocrystals. Because the same amount of the drug $(100 \mu \mathrm{g} / \mathrm{mL})$ was tested in the two groups, Taxol clearly had much more drugs solubilized in the culture medium. The quantitative uptake results of the nanocrystals confirmed the significant fluorescence observed inside KB cells by confocal microscopy at $3 \mathrm{~h}$ (Fig. 3). Moreover, the uptake was further examined varying the drug concentration of the two formulation groups (Fig. 7). No significant changes in drug concentration were detected intracellularly for the Taxol group, and the relative amount decreased from 2.02 to $0.34 \%$, when the total drug treatment went from 12.5 to $100 \mu \mathrm{g} / \mathrm{mL}$. Most of the drug was still found outside the cells in the media. Conversely, the cellular uptake of PTX-NCs was significantly promoted as the drug dose increased, suggesting

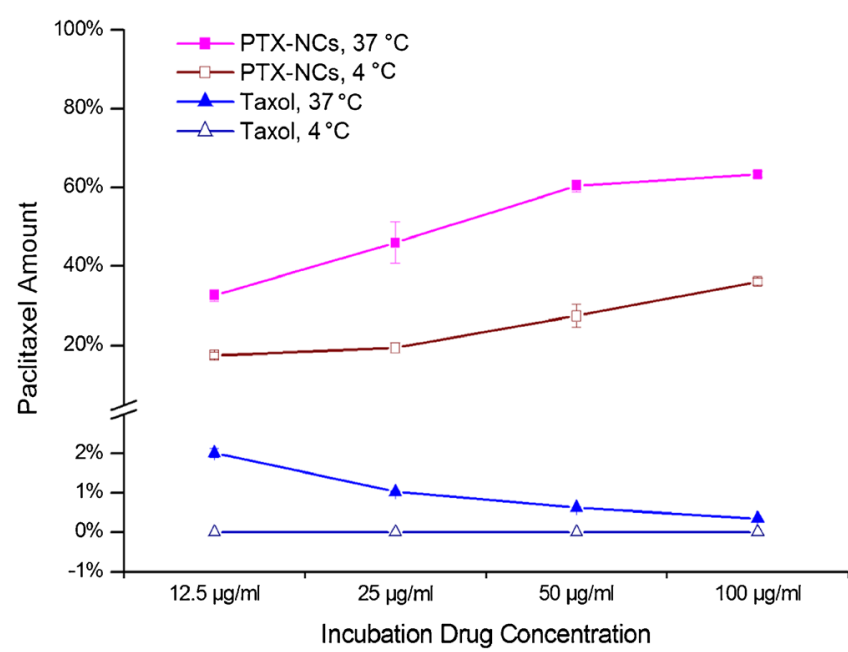

Fig. 8. Percentage of dosed PTX internalized in KB cells of Taxol (soluble PTX) and nanocrystals (PTX-NCs) at 4 and $37^{\circ} \mathrm{C}$. The incubation was $3 \mathrm{~h}$

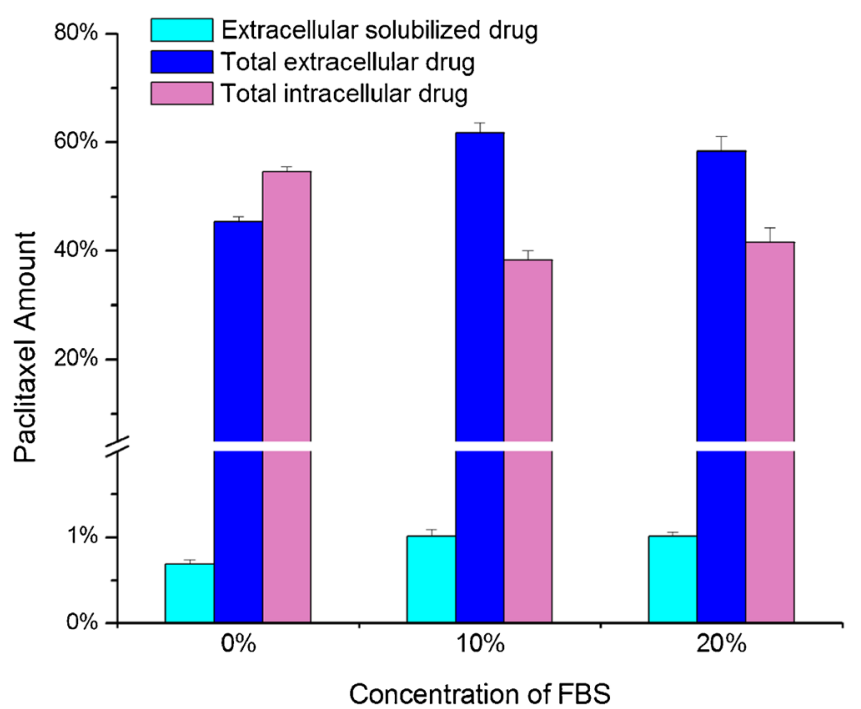

Fig. 9. Cellular uptake measurement of PTX in KB cells of drug nanocrystals (PTX-NCs) at different FBS concentrations, 0, 10, and $20 \%$ ( $v / v$ of culture medium). The incubation was $3 \mathrm{~h}$ and the total drug treatment was $100 \mu \mathrm{g} / \mathrm{mL}$. Intracellular and extracellular drug concentrations were measured

direct cellular uptake of the nanocrystals and echoing the confocal imaging results shown in Fig. 4.

The temperature effect on cellular uptake of drug nanocrystals was investigated to further probe the uptake mechanism. As shown in Fig. 8, the intracellular drug concentrations were at least twice at $37^{\circ} \mathrm{C}$ as those at $4^{\circ} \mathrm{C}$ when the cells were given PTX-NCs. The trend applied to all of four treatment concentrations, but it started to level off at the higher temperatures suggesting uptake saturation by the cells. The temperature-dependent internalization indicates endocytosis, which requires energy consumption $(11,12)$, responsible for the

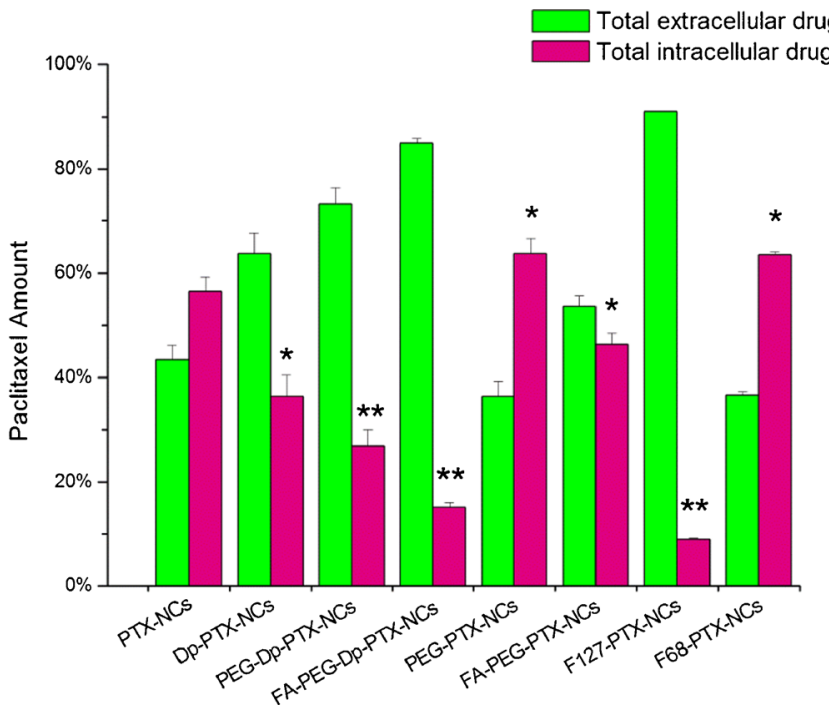

Fig. 10. Cellular uptake measurement of PTX in KB cells of pure and polymer-treated drug nanocrystals. The incubation was $3 \mathrm{~h}$ and the total drug treatment was $25 \mu \mathrm{g} / \mathrm{mL}$. Drug concentrations were measured intracellularly and extracellularly. * denotes significant difference between the marked group and pure PTX-NCs group $(P<0.05)$, and $* *$ denotes extremely significant difference $(P<0.01)$ 
nanocrystal uptake. For the Taxol group, the intracellular drug concentrations at $4{ }^{\circ} \mathrm{C}$ were too low to be detected. Solubility decrease at the lower temperature might attribute to the insignificant cellular uptake. The drug concentrations at $37^{\circ} \mathrm{C}$ are still no more than $2 \%$ for the concentration range tested.

Serum proteins are known to bind to hydrophobic drugs affecting biodistribution and pharmacokinetics $(13,14)$. To investigate possible influence on the cellular uptake of PTX nanocrystals, two different concentrations of FBS (fetal bovine serum) were tested in cell culture media and drug internalization was measured and compared those without using FBS (Fig. 9). In the presence of FBS, about $15 \%$ decrease in drug concentration was measured intracellularly. Slight increase in the solubized drug was also observed extracellularly in the media. However, there is no significant difference in cellular uptake $(P>0.05)$ between the two groups treated by 10 and $20 \%$ FBS. It is thus suspected that some of drug particles or free drug molecules might bind to serum proteins such as albumin, limiting the cellular uptake of drug nanocrystals to some extent.

As illustrated by the confocal imaging studies, polymer treatment, either by surface coating or physical integration, can have a profound impact on the cellular uptake of paclitaxel nanocrystals. Figure 10 shows drug internalization of polymer-treated nanocrystals relative to that of pure PTXNCs. All polydopamine-treated groups exhibited considerably reduced cellular uptake. The PEG treatment further decreased the intracellular drug concentration while FAPEG-Dp-NCs showing the smallest uptake, which is about half of concentration by Dp-PTX-NCs and $1 / 3$ by PTX-NCs. Moreover, the physical integration of $\mathrm{PEG}$ into drug nanocrystals indeed enhanced the drug internalization; the FA-PEG integration, however, negated the trend. Of the two surfactant-coated groups, $\mathrm{F}_{127}$ yielded the lowest intracellular drug concentration among all the nanocrystal groups. The $\mathrm{F}_{68}$-coated nanocrystals, interestingly, promoted additional cellular uptake of drug particles. These results support the confocal microscopic observations (Fig. 5).

In conclusion, our cellular uptake studies of paclitaxel nanocrystals in KB cells explored cellular uptake mechanism of drug nanocrystals. By utilizing confocal imaging and quantifying drug internalization kinetics, our in vitro investigation supports the notion that the drug nanocrystals could be taken up directly by the cells as solid particles. The intracellular drug concentrations were much higher than those of the solubilized drug formulation (Taxol); more than half of co-cultured drug nanocrystals were taken up by $\mathrm{KB}$ cells, as compared to about $2 \%$ of Taxol. The confocal imaging and temperature-dependent internalization results indicate that endocytosis may be responsible for the nanocrystal uptake. In addition, our studies examined polymertreated nanocrystals and the impact on drug internalization by the polymer coating or physical integration to the nanocrystals. While no clear trend can be identified, the polymer treatment exhibited significant influences, both positive and negative. All polydopamine-treated nanocrystals including FA conjugation drastically reduced the intracellular drug concentrations. And among the physical integration and surfactant adsorption, the nanocrystals incorporated with PEG and coated with $\mathrm{F}_{68}$ enabled additional cellular uptake compared with pure PTX-NCs. The $\mathrm{F}_{68}$-treated PTX-NC formulation is currently tested in vivo.
The cellular uptake studies provide further support to our pursuit of using drug nanocrystals for delivering poorly soluble drugs. The nanocrystal formulation of a chemotherapeutic drug can exert sufficient and likely stronger therapeutic effects against cancer cells than conventional, solubilized delivery systems. As a drug nanocrystal slowly dissolves inside a cell, the drug concentration builds up intracellularly, achieving a much lethal microenvironment for the cell, which may be difficult to achieve by solubilized delivery systems via drug diffusion through cell membrane. Undissolved nanocrystals can be further recycled by other cells. Our preliminary cytotoxicity results suggest that drug nanocrystals are much more potent than solubilized drug formulations in the KB cells (data not shown and to be published elsewhere). Direct cellular uptake and intracellular drug release thus make nanocrystal formulations appealing for cancer therapy.

\section{ACKNOWLEDGMENTS}

This work was financially supported by Lilly Endowment Seed Grant Program.

\section{REFERENCES}

1. Hollis CP, Weiss HL, Leggas M, Evers BM, Gemeinhart RA, Li T. Biodistribution and bioimaging studies of hybrid paclitaxel nanocrystals: lessons learned of the EPR effect and imageguided drug delivery. J Control Release. 2013;172(1):12-21. doi:10.1016/j.jconrel.2013.06.039.

2. Hollis CP, Weiss HL, Evers BM, Gemeinhart RA, Li T. In vivo investigation of hybrid paclitaxel nanocrystals with dual fluorescent probes for cancer theranostics. Pharm Res. 2014;31(6):14509. doi:10.1007/s11095-013-1048-x.

3. Seo K, Chung SW, Byun Y, Kim D. Paclitaxel loaded nanoaggregates based on $\mathrm{pH}$ sensitive polyaspartamide amphiphilic graft copolymers. Int J Pharm. 2012;424(1-2):26-32. doi:10.1016/ j.ijpharm.2011.12.047.

4. Zhou H, Liu X, Guo X, Li N, Yu W, Zhang Y, et al. Synthesis and characterization of amphiphilic chitosan derivatives as a nano-carrier for paclitaxel delivery. J Control Release. 2011;152 Suppl 1:e124-5. doi:10.1016/j.jconrel.2011.08.166.

5. Yang T, Cui FD, Choi MK, Lin H, Chung SJ, Shim CK, et al. Liposome formulation of paclitaxel with enhanced solubility and stability. Drug Deliv. 2007;14(5):301-8. doi:10.1080/ 10717540601098799.

6. Tao Y, Han J, Wang X, Dou H. Nano-formulation of paclitaxel by vitamin $\mathrm{E}$ succinate functionalized pluronic micelles for enhanced encapsulation, stability and cytotoxicity. Colloids Surf B: Biointerfaces. 2013;102:604-10. doi:10.1016/j.colsurfb.2012.08.062.

7. Shahin M, Lavasanifar A. Novel self-associating poly(ethylene oxide)-b-poly(epsilon-caprolactone) based drug conjugates and nano-containers for paclitaxel delivery. Int J Pharm. 2010;389(12):213-22. doi:10.1016/j.ijpharm.2010.01.015.

8. Zhao R, Hollis CP, Zhang H, Sun L, Gemeinhart RA, Li T. Hybrid nanocrystals: achieving concurrent therapeutic and bioimaging functionalities toward solid tumors. Mol Pharm. 2011;8(5):1985-91. doi:10.1021/mp200154k.

9. Gullotti E, Park J, Yeo Y. Polydopamine-based surface modification for the development of peritumorally activatable nanoparticles. Pharm Res. 2013;30(8):1956-67. doi:10.1007/s11095013-1039-y.

10. Ho CC, Ding SJ. The pH-controlled nanoparticles size of polydopamine for anti-cancer drug delivery. J Mater Sci Mater Med. 2013;24(10):2381-90. doi:10.1007/s10856-013-4994-2. 
11. Decuzzi P, Ferrari M. The role of specific and non-specific interactions in receptor-mediated endocytosis of nanoparticles. Biomaterials. 2007;28(18):2915-22. doi:10.1016/ j.biomaterials.2007.02.013.

12. Mamdouh Z, Giocondi MC, Laprade R, Le Grimellec C. Temperature dependence of endocytosis in renal epithelial cells in culture. Biochim Biophys Acta. 1996;1282(2):171-3. doi:10.1016/0005-2736(96)00077-6.
13. Ernsting MJ, Murakami M, Roy A, Li SD. Factors controlling the pharmacokinetics, biodistribution and intratumoral penetration of nanoparticles. J Control Release. 2013;172(3):782-94. doi:10.1016/j.jconrel.2013.09.013.

14. Aggarwal P, Hall JB, McLeland CB, Dobrovolskaia MA, McNeil SE. Nanoparticle interaction with plasma proteins as it relates to particle biodistribution, biocompatibility and therapeutic efficacy. Adv Drug Deliv Rev. 2009;61(6):428-37. doi:10.1016/j.addr.2009.03.009. 